\title{
LAS ISLAS FILIPINAS Y LA ETAPA FORMATIVA DE LA CONSTRUCCIÓN NAVAL ESPAÑOLA EN ASIA (1519-1657)
}

\author{
IVÁN VALDEZ-BUBNOV \\ Universidad Nacional Autónoma de México
}

\begin{abstract}
RESUMEN. El objetivo del presente estudio es comprender la etapa formativa de la industria de la construcción naval española en Asia, a partir de la percepción oficial acerca de la riqueza y la utilidad de los recursos madereros existentes en las islas Filipinas, así como de los mecanismos desarrollados para la movilización de recursos, humanos y forestales, durante este período formativo, y sus consecuencias sociales y políticas. Se propone también confrontar la legislación promulgada para normar la movilización de mano de obra para el uso de recursos forestales, los testimonios al respecto de la calidad y utilidad náutica de los distintos tipos de maderas y, finalmente, las manifestaciones de la tensión generada por la construcción naval entre las instituciones constitutivas del orden colonial en Filipinas durante el siglo XVI y la primera mitad del siglo XVII.
\end{abstract}

Palabras clave: maderas, construcción naval, sureste asiático, Filipinas, encomienda.

\begin{abstract}
AbSTRaCr. The purpose of this study is to understand the formation of the Spanish shipbuilding industry in the south Pacific during the sixteenth century. It deals with early Spanish assesments of the timber resources of the Philippines; the shipbuilding expertise of the local populations; their ability to produce essential manufactures, and the importance of the Spanish access to the South-East Asian intertional market. It also seeks to explain the social consequences of forced labour, and the negative impact it seemed to have in the official perception of the quality of Philippine shipbuilding timbers. It also examines the legislation issued by the crown to deal with these problems, and its interaction with the Spanish religious orders established in the islands during the first half of the seventeenth century.
\end{abstract}

Keywords: timber, shipbuilding, Philippines, Southeast Asia, encomienda.

Recibido: 11-3-2019 • Aceptado: 16-9-2019 • ivanvaldezbubnov@yahoo.com 
EL obJetivo del presente estudio es esbozar el desarrollo de la industria de la construcción naval española en Asia durante los siglos XVI y XVII, a partir de una doble perspectiva. Por un lado, se trata de la percepción oficial que se tenía acerca de la riqueza y utilidad de los recursos forestales de las islas Filipinas, la cual pasó desde una relativa indiferencia, derivada de las expectativas iniciales del tráfico de especias, a constituir un aspecto central de la exaltación de la naciente industria de construcción naval. Por otro lado, se trata de comprender los mecanismos desarrollados para la movilización de recursos, humanos y forestales, en este período formativo, así como sus consecuencias sociales y políticas. En efecto, la calidad de las maderas filipinas ha sido considerada por la historiografía reciente como uno de los factores que llevaron a diversos oficiales de la corona a sugerir la transferencia de la industria de la construcción naval desde el archipiélago hacia otras regiones asiáticas, como Cochinchina, Camboya y Siam (Prieto, 1984; McCarthy, 1995; Bankoff, 2004; Sales-Colín, 2013; Valdez-Bubnov, 2017).

La hipótesis central del presente trabajo es que, si bien la correspondencia oficial presenta menciones recurrentes al pudrimiento de maderas en la fabricación de las distintas clases de buques, esto no equivale a la existencia de una corriente de opinión basada en una percepción negativa de la calidad de las maderas filipinas. La causa del progresivo cambio de actitud hacia las ventajas de la construcción naval en el archipiélago, paralelo al desarrollo de esta industria durante los primeros treinta años de la colonia, se encuentra, en cambio, en otros factores. Se trata de la tensión social provocada por los mecanismos para la movilización de fuerza de trabajo para la boga de galeras y cortes madereros, así como de la fricción entre las estructuras administrativas y sociales del sistema colonial: la administración pública, la encomienda, y las órdenes religiosas. Por ende, el presente estudio se propone confrontar la legislación promulgada para normar la movilización de mano de obra para el uso de recursos forestales, los testimonios al respecto de la calidad y utilidad náutica de los diversos tipos de maderas y, finalmente, las manifestaciones de la tensión generada por la construcción naval entre las instituciones constitutivas del orden colonial en Filipinas.

\section{La primera expansión española en el Pacífico y la percepción de sus recursos náuticos}

La primera narrativa de la exploración española del Pacífico se debe a la pluma de Antonio de Pigafetta, cronista de la expedición de Magallanes (1519-22), quien describió copiosamente la flora de las islas visitadas en su recorrido, especialmente, la presencia y densidad de las forestas de árboles de clavo, canela, pimienta y nuez moscada, aunque sin prestar una particular atención a los recursos náuticos (Pigafetta, 
2004). Este relato, sin embargo, fue un punto de referencia esencial para los autores que, durante las décadas siguientes comenzaron a describir los recursos naturales de las islas del Pacífico y, como parte de ello, su potencial para el establecimiento de una industria regional de construcción de navíos. En efecto, la narrativa de Pigafetta aparece incorporada, treinta años después de su conclusión, en la primera historia global de la expansión ibérica del siglo XVI: el Libro XX, Segunda Parte, de la General Historia de las Indias de Gonzalo Fernández de Oviedo. Aunque la atención dedicada a los recursos forestales en esta obra, al igual que en la de Pigafetta, se limita a los árboles de especias, particularmente en las Molucas, el modelo narrativo basado en la descripción geográfica, seguida por los recursos naturales susceptibles de explotación, se convirtió en una constante en las obras, no sólo de los cronistas e historiadores del Pacífico hispano, sino también de los frailes misioneros que, posteriormente, relataron la historia de la conquista de las Filipinas y las relaciones diplomáticas de la corona española con los reinos de China, Ternate, Tidore, Champa, Camboya y Siam (Martínez Shaw, 2001, pp. 3-10).

Por otra parte, es importante señalar que, aunque el interés inmediato, tanto de Pigafetta como de Oviedo, se hallaba en la abundancia y calidad de los árboles de especias en las regiones visitadas y descritas, la compleja narrativa histórica del segundo presenta, también, un atisbo de la importancia que pronto adquirieron los recursos forestales del Pacífico, no ya para el mero tráfico de clavo, pimienta, canela y nuez moscada, sino para la articulación de un comercio regular de manufacturas y materias primas entre las economías desarrolladas del lejano oriente y la Nueva España y el Perú, a través de las islas Filipinas. En efecto, el relato de Oviedo señala tres puntos de importancia estructural para el establecimiento de la presencia española en Asia: la pericia técnica de las poblaciones locales en la construcción de embarcaciones, la presencia de forestas con abundantes y diversas maderas para diferentes usos, y la existencia de activos mercados capaces de proporcionar materiales de construcción naval, especialmente la clavazón de hierro. Estos puntos dan pie, en esta obra, a una minuciosa descripción técnica de las embarcaciones de cabotaje empleadas por los nativos de Ladrones (Marianas). Esta descripción, aunque de orden casi etnográfico, es un indicio relevante de la atención dedicada por observadores subsecuentes a las técnicas de construcción naval de las poblaciones del Pacífico sur. Es como sigue:

las canoas en que andan son de quatro y cinco brazas de luengo y mayores y menores y angostas que tendrán de ancho dos codos o poco menos, son algunas de una pieza y otras de muchas, y tienen sendos contrapesos... de una madera hecho como una Toninna quasi del largor de la mitad de la canoa la qual amarrada fuertemente en dos palos que sale de la canoa apartada del cuerpo d'ella obra de una braza, y tanto andan sobre la popa como sobre la proa, no ay diferencia de la popa 
a la proa, tienen velas latinas de esteras muy bien texidas y para hacer otra buelta no buelven la canoa, sino buelven solamente la vela y hazen de la popa proa y de la proa popa quando quieren, son estas canoas de altor hasta la rodilla de un hombre, y las tablas pegan unas con otras d'esta manera, que horádanlas en los bordes de las tablas y atan las unas con las otras con unas cuerdas que hazen de cortezas de árboles, y por la parte de dentro dexan unos pedazos de madera horadados sobre los quales atraviesan unos palos que amarran para fortificarlas, y por de fuera las brean con un betún que hazen de cal y azeite con que betunan y cierran todas las costuras, de forma que no haze agua (Fernández de Oviedo, 1557, fol. 36).

El autor nos ofrece también las dos primeras menciones explícitas a la construcción naval española en Asia. La primera fue la orden dada en 1527 por el capitán general Martín Íñiguez de Carcaño para construir una nao en Tidore, con el fin de enviarla a España cargada de clavo y especias. La capitana de su expedición estaba averiada y, además, era preciso informar al emperador de la situación en las islas de la especiería, y de la guerra que allí se tenía con los portugueses (Fernández de Oviedo, 1557, fol. 44). Es importante señalar, sin embargo, que la mención a este barco posee un antecedente preciso en la temprana literatura de viajes al Pacífico, anterior incluso a la publicación del libro de Oviedo, específicamente, en la obra de García de Escalante Alvarado, factor de la expedición de Ruy López de Villalobos a las Molucas (1542). Esta relación fue escrita en Lisboa, en 1548, y es actualmente conocida por la historiografía como Viaje a las islas del Poniente. En ella se señala que el rey de Tidore, cuyo antecesor inmediato había dado hospitalidad a las expediciones de Magallanes y Loaysa (1525-1536), ofreció a los castellanos de Villalobos, en competencia con los portugueses, ayuda para construir:

una nao tan grande y tan buena que fuese suficiente para descubrir el camino de la Nueva España. Y para ello daría carpinteros de la tierra que ayudasen a los nuestros, y toda la madera y tablazón y brea y jarcia de la tierra [...] pues ya había ayudado (a los portugueses) a hacer una nao de trescientos toneles, que mejor lo haría agora siendo para servir a Su Majestad (Escalante Alvarado, 2015, p. 82).

Esto significa que la construcción naval portuguesa en las Molucas, derivada de su establecimiento allí en 1512 (en alianza con el sultanato de Tidore contra de sus rivales de Ternate), era un hecho incluso antes de la llegada de la expedición de Loaysa $\mathrm{y}$, aunque la fecha de construcción de aquél buque portugués es incierta, bien puede ser anterior incluso a la presencia de Magallanes y Pigafetta. En todo caso, esta es la primera mención explícita en la literatura española de la exploración del Pacífico acerca de la posibilidad de contar con el apoyo de centros de poder regionales para la movilización de recursos de construcción naval. Por otra parte, los buques que constituyeron 
las «armadas de la especiería» fueron construidos, ya en la Península Ibérica — caso de la armada de García Jofre de Loaysa (1525) - ya en las costas de América central — caso de las armadas de Álvaro de Cerón Saavedra (1527), de Hernando de Grijalva (1537) y, por supuesto, de Ruy López de Villalobos (1542)—. Los buques que debían constituir las nonatas expediciones de Simón Alcazaba y Pedro de Alvarado también fueron construidos en esa región, mientras que Hernán Cortés construyó varios para una expedición transpacífica (Álvaro de Cerón Saavedra, 1527), y cuatro expediciones exploratorias de las costas de Nueva España entre 1532 y 1539 (Brand, 1956; Radell, 1971; Rubio, 1977; Clayton, 1980; Sánchez, 2001; Ortuño, 2005).

En la obra de Oviedo existe una segunda mención a la construcción naval española en Asia. Se trata de un buque alternativamente llamado «fusta» y «navío», que los castellanos comenzaron a construir en la isla de Gilolo, Molucas, y que los portugueses intentaron destruir con granadas de pólvora cuando sólo le faltaba el calafateo para ser botado al agua. Aunque el buque casi no recibió daños, no resulta claro si efectivamente fue utilizado, pues, según Oviedo, «como los castellanos eran nuevos en aquella tierra, no supieron conocer la madera, y salió tan mala que al tiempo que la quisieron calafatear la hallaron quasi toda podrida» (Fernández de Oviedo, fol. 47). Hay cierta ambigüedad en el texto, pues posteriormente se señala que en la Navidad de 1527 «se botó la fusta de los castellanos al mar y la llevaron a Tidore desde Gilolo, donde se hizo» (Fernández de Oviedo, fol. 49). En todo caso, es probable que haya tenido lugar, si no aquella, alguna otra botadura en la región, pues en 1528, cuando Andrés de Urdaneta salió de Tidore para alcanzar al galeón Florida, venido de la Nueva España bajo el mando de Álvaro de Cerón Saavedra, lo hizo en algunas embarcaciones de remo, construidas allí, y bogadas por naturales (Peterson, 2014, p. 83). En todo caso, los pasajes de Oviedo a este respecto son importantes porque reflejan una temprana toma de conciencia de las posibilidades de la construcción naval en los reinos del Pacífico, aparejada con una constatación tácita de las dificultades de su puesta en práctica, particularmente en relación con el desconocimiento de la calidad de las maderas locales.

El tráfico español de especias disminuyó en importancia tras la renuncia del emperador a continuar disputando las Molucas a Portugal, y el fracaso de las ya mencionadas expediciones (Giraldez, 2015, pp. 37-38). Todo ello, sumado a la falta de una ruta marítima estable entre América y Filipinas, retrasó la consolidación de la presencia española en Asia. Así, no resulta sorprendente que las evaluaciones de los recursos para la construcción naval sean escasas en los relatos de exploración de este período. Tal es el caso del escrito de Juan de la Isla, titulado Descripción y Relación [...] de los Puertos de Acapulco y Navidad, y de las Islas [...] del Poniente, fechado en 1564, el cual ofrece detalles precisos acerca de la locación y geografía del archipiélago filipi- 
no, así como de las costumbres y dieta de sus moradores. Salta a la vista la ausencia de referencias a las posibilidades de la industria de construcción naval (aunque presta atención a las características de las naves de los moros de Mindanao), pese a que el propósito del manuscrito era obtener de la corona dos navíos de 250 toneladas, artillería y ochenta soldados, para llevar a cabo la «conquista y contratación» de las islas ${ }^{1}$.

\section{El nacimiento de la industria de la construcción naval española en Filipinas}

No sería sino hasta 1565, con el descubrimiento del tornaviaje por la expedición de Legazpi-Urdaneta, cuando se estableció un tráfico regular entre Nueva España y el archipiélago filipino. Las constantes llegadas y salidas de buques novohispanos iniciadas a partir de ese año hicieron evidente la necesidad inmediata de contar allí con una infraestructura permanente de apresto y construcción naval y, al menos desde 1567, existen indicios de que el establecimiento de un astillero en Filipinas era considerado como una necesidad de primer orden (Sales-Colín, 2013, p. 59). De hecho, Miguel López de Legazpi, conquistador de Filipinas, en una carta enviada a Felipe II en 1568, elogió las forestas de esas islas y su potencial para la construcción naval (Bankoff, 2004, p. 323; Wing, 2015, p. 113). Existían buenas razones para ello. En 1566, el galeón San Jerónimo arribó desde Acapulco transportando muy escasos socorros para la naciente colonia española. El buque llegó tan comido de broma que, según el testimonio de su piloto, hubiera sido necesario cambiarle la quilla, mientras que las tracas se separaban por tres dedos, y tomaba más agua de la que era posible vaciar. Pese a los esfuerzos por calafatearlo, tuvo que ser dado al través (Peterson, 2014, p. 79). Claramente, los daños provenían de la larga travesía del Pacífico, particularmente, de los efectos corrosivos de la salinidad en la clavazón de hierro. Más aún, las aguas del río Pásig, que desembocan en la bahía de Manila, estaban infestadas de teredo navalis — el parásito causante de la broma (Peterson, 2014, p. 80) — . Todo ello constituyó un estímulo directo para la utilización de embarcaciones locales con el objetivo de completar la exploración de las Visayas. En 1570, Legazpi envió a Martín de Goiti a Luzón con quince de ellas. También existen indicios de que Legazpi intentó construir seis galeras, las cuales habrían representado el primer caso de construcción naval española en Filipinas (Amaya, 2017).

La fundación de Manila en 1571 fue seguida del primer esfuerzo oficial para resolver el problema de la infraestructura naval del archipiélago. Se trató de la salida desde Lisboa de una armada de socorro, bajo el mando de Antonio de la Torre, con

Museo Naval de Madrid (en adelante MNM), Colección Fernández de Navarrete, XVII, 322. 
diversos materiales de calafateo y reparación, e instrucciones específicas de reconocer los parajes para el establecimiento de un astillero, y evaluar tanto la pericia técnica como la abundancia de la mano de obra indígena. Esto hizo posible resolver la urgencia inmediata de efectuar carenas y recorridas a las naos Santiago y San Juan, en el año de 1572, para su retorno a Acapulco (Sales-Colín, 2013, p. 60). El financiamiento de la construcción de navíos por cuenta de la Real Hacienda, por otra parte, quedó formalizado con las instrucciones de navegación y comercio expedidas entre $1571 \mathrm{y}$ 1573, las cuales también estipulaban el número de unidades en servicio para la ruta Manila-Acapulco y la cadencia de sus salidas en ambas direcciones (Valdez-Bubnov, 2017, p. 229-230).

Es preciso considerar que la ruta de Manila-Acapulco no fue la única fuente de demanda de navíos, insumos navales e infraestructuras de mantenimiento. La ambición de usar las Filipinas como base para la expansión a China, seriamente considerada por el segundo gobernador, Guido de Lavezares, así como la embajada de los frailes Agustinos Martín de Rada y Jerónimo Martín, enviada a ese reino en 1575, operaron en un sentido análogo (Cervera, 2013; Ostolaza, 2006). Esto explica la creciente atención a los recursos forestales en la correspondencia recibida por Felipe II durante estos años. En 1574, Andrés de Mirandola señalaba la existencia de «maderas incorruptibles», como «ébanos, cipreses y cedros» (Bankoff, 2004, p. 322). En 1575, Juan Pacheco Maldonado estimaba que, dada la riqueza maderera de las islas, debía ser posible producir de tres a cuatro galeones cada año (Bankoff, 2004, p. 323; Wing, 2015 , p. 113). En un sentido convergente, la obra fundacional de la etnografía filipinista, la Relación de las Islas Filipinas y de lo que en ellas han trabajado los padres de la Compañia de Jesús, de fray Pedro de Chirino (impresa en 1604), la cual narra sucesos correspondientes a la etapa fundacional de la colonia española, contiene la primera denominación precisa de la principal «madera incorruptible» de Filipinas, el molaui, usada en la construcción de la capilla de la iglesia de Manila, y frecuentemente empleada en la confección de ataúdes por la población local. También incluye una mención a la jarcia elaborada con la palma cauayan, y a su uso para la fabricación de bombas de achique de eficacia extraordinaria (Chirino, 1890, pp. 58 y 108).

Los estudios históricos que hasta ahora incluyen menciones a los tipos de maderas filipinas utilizadas durante este período, derivan todos del conocido informe del capitán de maestranza Sebastián de Pineda, fechado en Cavite en 1619 (Blair y Robertson, 1904, vol. XVIII, pp. 169-188). Se trata de un informe valioso, pero conocido, el cual constituye la base de las descripciones de las maderas filipinas en la historiografía. En el presente estudio, la nomenclatura y catálogo de maderas de construcción está basada en tres fuentes de períodos distintos, pero que, desde una perspectiva técnica, cubren su espectro temporal sin peligro de anacronismo, y que ofrecen una visión un 
poco más amplia. La primera es la Historia natural de las islas Bisayas (1668), del padre Francisco Alzina, la cual presenta observaciones forestales realizadas hacia 1620 (Yepes, 1996, pp. 129-142). Esta importante obra constituyó el modelo analítico e interpretativo de los recursos naturales del Pacífico español, según el paradigma pliniano previamente desarrollado por el padre José de Acosta en su Historia natural y moral de las Indias (Sevilla, 1590). La segunda fuente es el manuscrito anónimo de la Real Academia de la Historia titulado Descripción de las Yslas Philipinas, fechado en 1746 (Hidalgo, 2009a, pp. 38-40). La tercera, la constituyen los estudios forestales publicados por Vidal y Soler en el siglo XIX (Vidal, 1874, pp. 148-181).

En síntesis, las maderas usadas para quillas y codastes eran las nombradas Bétis, Dungon, e Ipil. Para curvas de consolidación, ligazones y rodas, se empleaba el molave. Para tracas y forros exteriores, la banaba. El guijo se empleaba para baos y arboladura. El batintinas, para sobrequillas y durmientes. El mangachupuy, para trancaniles y latas de cubierta. Para obras muertas y repartimientos de interiores, se utilizaba el amuguis de Mariveles. El palo María recibía diversos usos, según la forma del tronco, especialmente en ligazones, si era curvo, o en arboladura, si tenía secciones rectas. El siguiente cuadro presenta una clasificación general de maderas de construcción, ordenadas según sus propiedades físicas, de mayor a menor (Vidal, 1874. pp. 178-181; Cruz, 1963; Meniado, 1975; Cordero, 1991; Tamolang, 1995; Rojo, 1997 y 1999):

\begin{tabular}{|l|l|l|}
\hline Elasticidad & Resistencia & Peso específico \\
\hline Calantás & Pagatpat & Ébano \\
\hline Solipa & Bansalagui & Camagon \\
\hline Antípolo & Yacal & Yacal \\
\hline Lanete & Culing-manoc & Pagatpat \\
\hline Anagap & Manicnic & Anusep \\
\hline Baticulin & Ipil & Manicnic \\
\hline Apiton & Molave & Dungon \\
\hline Amuguis & Narra & Molave \\
\hline Macasin & Cubí & Teca \\
\hline Bancal & Guijo & Tíndalo \\
\hline Anubiong & Acle & Bolongita \\
\hline Marang & Ébano & Camayuan \\
\hline Calumpan & Camagon & Ipil \\
\hline Malarujat & Tíndalo & Pasac \\
\hline Calumpit & Calamansanay & Lanutan \\
\hline Banaba & Calumpan & Banaba \\
\hline Anusip & Anusip & Cubí \\
\hline Malatalan & Pino & Culing-manoc \\
\hline Manicnic & Palonapuy & Mangachapuy \\
\hline Mayapis & Panguisan & Calumpang \\
\hline Acle & Camayuan & Panguisan \\
\hline Calamansanay & Dungon & Bétis \\
\hline
\end{tabular}




\begin{tabular}{|l|l|l|}
\hline Narra & Bolongit & Acle \\
\hline Balao & Mangachapuy & Guijo \\
\hline Molave & Bétis & Macasin \\
\hline Guijo & Lanutan & Bansalagui \\
\hline Palonapuy & Antípolo & Calumpit \\
\hline Tíndalo & Bancal & Malatalan \\
\hline Cubí & Balao & Calamansanay \\
\hline Yacal & Malatalan & Malarujat \\
\hline Camayuan & Nato & Narra \\
\hline Santol & Banaba & Apiton \\
\hline Bolongita & Tangile & Pino \\
\hline Dungon & Palo-maría & Tangile \\
\hline Mangachapuy & Macasin & Antípolo \\
\hline Nato & Malarujat & Anubiong \\
\hline Teca & Pasac & Malacadius \\
\hline Malacadius & Mayapis & Nato \\
\hline Panguisan & Lanete & Palo María \\
\hline Pinto & Santol & Palonapuy \\
\hline Bétis & Anubiong & Calantás \\
\hline Ipil & Malacadius & Amuguis \\
\hline Ebano & Anagap & Bancal \\
\hline Camagon & Calumpit & Mayapis \\
\hline Bansalagui & Apiton & Baticulin \\
\hline Culing-manoc & Baticulin & Lanete \\
\hline Lanutan & Calantás & Anagap \\
\hline Pagatpat & Marang & Santol \\
\hline & Sulipa & Marang \\
\hline & & Sulipa \\
\hline & & Balao \\
\hline
\end{tabular}

Tal como en el caso de las forestas del archipiélago, la tecnología de la construcción naval y la habilidad artesanal de la población indígena fue elogiada por algunos observadores españoles, pero ninguno tan influyente como Antonio de Morga, quien, en su historia de las gubernaturas de este período, legó a la posteridad la descripción más completa de las embarcaciones de guerra de las poblaciones del archipiélago. Es la siguiente:

sus navíos y embarcaciones, son de muchas maneras [...] unas canoas de un palo, muy grandes, y de bancas, hechas de tablazón, armadas sobre quillas. Y de vireyes y barangayes, que son unos navíos muy sutiles y ligeros bajos de bordo, clavados con cavilla de madera, tan sutiles por la popa como por la proa, en que caben muchos remeros por ambas vandas, que con buzeyes o canaletes, y con gaones bogan por fuera del bordo [...] Encima de los remeros hay un Bailío o crujía, armada de cañas, sobre que anda la gente de pelea, sin embarazar la esquifazón de remeros, en que, conforme a la capacidad del navío, va el número de la gente; y desde allí, se marea la vela, que es quadrada y de lienzo, en una cabria, hecha de dos cañas 
gruesas, que sirve de árbol, y quando el navío es grande, lleva también trinquete, de la misma forma, y ambas cabrias, con sus encajes, para abatirlas sobre la crujía quando el viento es contrario, y sus timoneles en popa para governar. Lleva otra armazón de cañas, en la misma crujía, en la qual, quando haze sol o llueve, se arma una tienda de esteras, tejidas de hojas de palmas, muy espesas y tupidas, que llaman Cayanes. Con que todo el navío y gente de él, va cubierta y reparada. Va también hecha, otra armazón de cañas gruesas, por ambas vandas del navío, por todo lo largo de él, fuertemente atadas, que van besando el agua, sin que impidan la boga, que sirven de contrapesos, para que el navío no pueda trastornarse ni zozobrar, por mucha mar que aya, ni fuerza de viento que la vela lleve [...] Destos navíos, se usa comúnmente en todas las islas desde su antigüedad, y de otros mayores, que llaman caracoas, y lapis y de tapaques [...] Ay algunos tan grandes, que llevan cien remeros por banda, y treinta soldados [...] y los comunes son varangayes, y vireyes, de menos esquifazón y gente, y ay muchos de ellos, en lugar de la cavilla de madera, y costura de tablas, los clavan con clavazón de hierro, y los timones y las proas con espolón a la castellana (Morga, 1997, p. 258).

Este importante pasaje no deja ninguna duda respecto al elevado nivel técnico de la construcción naval indígena. También permite comprender su enorme potencial para la industria española, merced a la existencia de un artesanado experto en la labra de maderas para uso náutico. Sin embargo, la tecnología europea, sumada a las demandas de la navegación transpacífica, requerían una movilización de recursos mucho más intensa que las embarcaciones indígenas, lo que hizo que en esta etapa formativa se buscara abastecer a la naciente industria de insumos navales transportándolos, ya no desde la Península Ibérica, sino directamente desde Nueva España.

Las primeras instrucciones conocidas para la compra de manufacturas náuticas en Veracruz con destino a Manila fueron expedidas en 1576 por el segundo gobernador de las islas, Francisco de Sande, y preveían no sólo la adquisición de diversos artículos para la construcción de dos naos en torno a la bahía de Manila, sino también un presupuesto anual de sueldos para sus 170 tripulantes (Valdez-Bubnov, 2017, p. 229). Otro documento, enviado asimismo por Sande a México en relación a esta misma compra y contratación de operarios, ofrece indicios importantes acerca del nacimiento de la industria de la construcción naval española en Filipinas, a partir de la transferencia de recursos de Nueva España:

Dos maestros de hazer navíos y galeras. No los hay en esta tierra. Doze carpinteros de lo mesmo. Buscarse an acá, aunque se hallan con dificultad. Llevóse un carpintero, que no se pudo hallar más. Mandadores para galeras que sepan hazer vela latina. No los hay en esta tierra, si no viene alguno en esta flota. Cincuenta lombarderos. No los ay acá, sino los que están en el puerto, procurarse á de enviar algunos. Lleváronse cinco, y estos basta; que robles que fue por fundidor de artillería que avía servido 
toda su vida en estas casas reales mostrará [...] a los que de acá van [...] quando fuere menester. Dos maestros de fundir artillería. No los ay acá. Artillería gruesa, seis piezas de hasta quarenta quintales y dos cañones pedreros [que] será mejor que se funda en las yslas. Plomo [...] Lleváronse doscientos quintales, y esta es la primera vez que se a pedido [...] porque hasta ora nunca se a hecho allá ningún navío que aya de andar por esta carrera².

Como parte de este proceso de transferencia industrial, el 14 de mayo de ese mismo año, Sande promulgó las primeras ordenanzas para el gobierno de Filipinas, las cuales incluyen indicaciones precisas para la movilización de recursos náuticos. En efecto, el punto número 11, continuación de las instrucciones para la tasación y cobro de tributos a la población indígena, señalaba que los oficiales encargados de ello debían llevar registros de cuenta y razón de «carros de obra, tablas, estopa, brea e clavazón, e las demás cosas [...] para la obra de navíos, y galeras que se hazen e hizieren de su Magestad en los astilleros de estas yslas». De igual forma, estipulaba exactamente la compensación que los naturales empleados en las labores de astillero debían recibir por sus servicios a la corona: «si fueren menester yndios o moros carpinteros, o de otros oficios [...] los dichos oficiales les den y les paguen por cada quince días de los que sirvieren dos mays de oro, esto comiendo los dichos yndios a su costa, y cada quince días muden los dichos yndios officiales» (Morga, 1997, p. 52).

Este conjunto de medidas hizo posible iniciar la construcción naval en el archipiélago. Como hemos señalado, las primeras instrucciones de Sande para la transferencia de pertrechos y operarios desde Nueva España incluían, específicamente, dos maestros en la construcción «de navíos y galeras [...] porque no los hay acá», lo cual, evidentemente, indica que antes de esa operación no se construyeron otros buques españoles en Filipinas. Es altamente probable que los primeros hayan sido las embarcaciones usadas por la expedición de Esteban Rodríguez de Figueroa contra Joló y Minanado, en 1576, o las cuatro galeras y tres galeotas, destinadas a la expedición de Borneo de 1577 (Amaya, 2017)3. De ser así, es preciso considerar que, ante la ausencia de operarios españoles, estas puedan haber sido variantes de las embarcaciones tradicionales de la región.

En todo caso, las medidas administrativas tomadas por Sande hicieron posible construir los primeros dos navíos propiamente filipinos, bautizados Santísima Trinidad y Santa María de Jesús, ambos de 500 toneladas, los cuales fueron botados en astilleros temporales situados en Cavite y Otón durante los años de 1579 y 1580, respectivamente. En 1582, Juan Bautista Román, factor de la Real Hacienda en Manila,

Archivo General de Indias, Sevilla (en adelante AGI), Filipinas, I, I, 2/24.

AGI, Filipinas, 6, r. 3, n. 30. 
testificó acerca de la extrema baratura de las dos naves construidas por Francisco de Sande: «salieron muy baratas por la abundancia de maderas y porque los navíos de China traen hierro a muy buen precio» (Wing, 2015, p. 114). Esto consolidó del todo la transferencia de la industria, como lo manifestó el virrey Conde de la Coruña en 15844. La obra del padre Chirino, por otra parte, nos da un indicio de la proporción de éstas economías, en relación con la fábrica de Nueva España:

Para la fábrica de las [naos] hay tanta abundancia de los materiales y artífices necesarios, que una nao que en Nueva España ó Perú tiene de costo cincuenta y sesenta mil pesos y se tarda en fabricar algunos años, en Filipinas, se hace en menos de uno y con menos costo de ocho mil (Chirino, 1890, p. 17).

En 1580 tomó posesión de la gubernatura Gonzalo Ronquillo de Peñalosa, quien formalizó una fuente de manufacturas para la construcción naval en Filipinas, la cual muy pronto sustituyó casi enteramente el recurso de solicitarlas desde Nueva España. Se trata de la reglamentación y sistematización del comercio de importaciones de China y, en menor grado, también de Japón, Siam y Camboya, establecido en Manila casi desde la llegada misma de los españoles. Este comercio fue tan importante para la supervivencia de la colonia española, que el gobernador «les hizo alcaycería y parián dentro de la ciudad» (Morga, 1997, p. 57). Los productos chinos recibidos en Manila quedaron, a partir de este punto, gravados al 3\% de almojarifazgo, mientras que aquellos que eran reexportados a la Nueva España lo eran al 2\%. También se impuso un flete de doce pesos por tonelada de mercancía embarcada (Morga, 1997, p. 62). Esto coincidió con un incremento en la demanda de navíos, debido a la apertura del comercio directo con Perú; la colonización de la isla de Panay, en Otón; la pacificación y colonización de Cagayán; la exploración del sur del archipiélago en busca de nuevas rutas de salida a Nueva España ; y, especialmente, la armada dirigida contra Ternate por Juan Ronquillo, en 1582, para la cual se aprestaron «tres navíos grandes, y gran número de otros menores» (Argensola, 1609, p. 167-169), requiriendo, además, una fuerte transferencia de pertrechos a la guarnición española desplegada en la vecina isla de Tidore (Morga, 1997, p. 58).

La demanda de navíos de distintos portes para la pacificación de las Filipinas continuó con intensidad durante la gubernatura de Santiago de Vera (1584-89). Durante el primer año de su mandato, ordenó construir varias galeotas financiadas por los colonos, que pueden haber sido las usadas para la expedición de Juan Morón contra Ternate. Durante el último año, se construyeron cuando menos dos navíos, tres galeras y un número indeterminado de embarcaciones menores (Amaya, 2017). En

AGI, Filipinas, 339, 1. 1. 
este período, por otra parte, dio inicio la presión de rivales europeos sobre la industria de construcción naval filipina, cuando el corsario inglés Cavendish capturó uno de los últimos galeones del Pacífico construidos en América central, el Santa Ana, para posteriormente atacar a un navío todavía en grada en Arévalo, Panay, sin lograr incendiarlo, gracias a la resistencia de la población local y de los oficiales españoles a cargo de la fábrica (Morga, 1997, p. 70).

En 1592 había en servicio cuando menos cuatro galeras armadas para el rechazo de piratas japoneses, y se preveía la construcción de otras más ${ }^{5}$. La demanda de navíos y galeras se intensificó a partir de este punto con las expediciones a Camboya de 1596, 1598 y 1603, y a las Molucas holandesas de 1606 (Argensola, 1609, p. 187). Por otra parte, la permanente guerra de corso contra los musulmanes de Mindanao operó en el mismo sentido, por lo que es probable que la producción de galeras hubiese sido mucho mayor que la documentada por la escasa historiografía existente (Barrantes, 2004, pp. 22-23).

Es posible que el término «galera», en este contexto, haya sido empleado para referirse de manera genérica a variantes de las embarcaciones de remo tradicionalmente construidas en la región, como las ya descritas caracoas, barangayes y vireyes. Esta sospecha se ve reforzada por que una de las características distintivas de la construcción naval indígena, como nos ha señalado Morga, era la posibilidad de evitar el uso de clavazón de hierro en la hechura del casco, empleando, en cambio, la técnica de costura de tracas, lo cual implicaba una reducida dependencia de las importaciones de hierro, novohispanas o asiáticas (Scott, 1982, pp. 335-376; Manguin, 2012, pp. 601-602). Cabe señalar que el uso de caracoas por los españoles, particularmente en las expediciones contra los moros de Mindanao, se encuentra bien documentado en este y otros períodos (Mallari, 1989; Peterson, 2014, p. 17).

Es importante considerar que el funcionamiento de la industria de construcción naval española en las Filipinas dependía no sólo de las importaciones, sino también de las manufacturas y la fuerza de trabajo indígena en Luzón y las Visayas. Las poblaciones locales poseían una importante producción de insumos necesarios para la fabricación de sus embarcaciones tradicionales, y algunas de ellas podían ser utilizadas en la construcción española. Se trata de la fibra de coco para calafateo, la jarcia de palma (abacá y gemú) para diversos aparejos, y las lonas de algodón hilado para el velamen. Estos productos podían ser obtenidos como parte del tributo cobrado en especie y, después de la monetarización, por medio de compras forzosas conocidas como vandalas, usualmente impuestas a precios inferiores al mercado, o sin mediar ninguna compensación inmediata (Hidalgo, 2009b, p. 47). Sin embargo, el elemento verdaderamente decisivo para la eficiencia económica de la industria era la posibilidad

AGI, Patronato, 263, n. 1, r. 5. 
de movilizar trabajadores indígenas para el corte o arrastre de maderas y, en principio, también para el labrado y ensamblado de las piezas del buque. La movilización de esta fuerza de trabajo fue motivo de fricciones sociales progresivamente graves, hasta el punto de que las responsabilidades técnicas de los trabajos de astillero fueron transferidas a especialistas chinos, con salarios comparativamente elevados, bajo la autoridad de oficiales españoles ${ }^{6}$.

El origen de estas fricciones se encontraba en la naturaleza del sistema desarrollado para el cobro de tributo y la movilización del trabajo indígena en América, la encomienda, el cual fue transferido a las islas Filipinas en una variante «suavizada», pero no por ello menos onerosa, o menos cruel (Hidalgo, 2009b, p. 43). Tal como en el caso de la encomienda americana, la desarrollada en Filipinas consistía en la cesión a un colono español del derecho a extraer de las poblaciones indígenas cargas fiscales definidas por tasación previa y pagadas, primero en especie y, a partir de 1589, en una tasa fija de diez reales (Phelan, 1959, p. 94; Hidalgo, 2001, p. 143). A cambio, el encomendero quedaba obligado a prestar servicio militar a la corona y participar en la defensa de sus encomendados; a asegurar su evangelización y proveer lo necesario para el ejercicio del culto; a mantener la productividad de la tierra y a residir en ella (Hidalgo, 2001, p. 90). La dimensión laboral de este sistema, el repartimiento, consistía en la movilización temporal y forzosa de la mano de obra indígena, conocida, en el caso de Filipinas como polo o servicios (Phelan, 1959, p. 99). Ambas vertientes del sistema estaban estrechamente ligadas al funcionamiento de la administración real en el archipiélago, la cual, conforme progresaba la conquista, fue dividida en gubernaturas regionales conocidas como Alcaldías Mayores: Manila, Bay, Pampanga, Pangasinán, Cagayán, Ilocos, Camarines, Tondo, Tabayas, Otón (Isla de Panay), Panay, Negros, Cebú, Leyte y Samar, Ybabao, Caraga (en Mindanao), Mindoro, Calamianes, Mariveles, Balayán y Bombón, Calilaya, Butuan, y Catanduanes (Hidalgo, 2009b, p. 35).

Los alcaldes mayores interactuaban con los encomenderos para canalizar los tributos o los productos obtenidos por vandala a las reales tesorerías o almacenes (según se tratase de recaudaciones en metálico o en especie), o directamente a los astilleros, (caso de tratarse de géneros para la construcción naval). También supervisaban los polos para obras públicas, particularmente las operaciones de corta y arrastre de maderas - conocidas específicamente como elas - o el servicio en los astilleros. Otra extendida forma de repartimiento en Filipinas eran las llamadas esquipazones o esquifazones, es decir, el reclutamiento para la boga de galeras y otras embarcaciones en servicio naval (Hidalgo, 2009b, p. 232). En todos los casos se trataba, en principio, de un trabajo remunerado, pues la corona había prohibido toda forma de esclavitud desde 1574.

AGI, Filipinas, 200, n. 206. 
Sin embargo, numerosos factores coincidían para hacer de la encomienda y el repartimiento una carga extremadamente gravosa para las sociedades indígenas de Filipinas. En efecto, ante la perpetua penuria de la Real Hacienda, numerosos oficiales reales padecían retrasos en sus salarios, lo cual les llevaba a acudir a las encomiendas como compensación y, en consecuencia, a explotarlas con mayor intensidad. Naturalmente, la compra de un cargo público operaba en el mismo sentido. En 1574, la corona había intentado hacer incompatible el ejercicio de funciones públicas con la posesión de una encomienda, pero los oficiales reales protestaron amenazando con abandonar sus cargos, para conservar sus encomiendas.

Entre 1576 y 1577, la corona formalizó la coexistencia de ambas funciones. Esto reforzó el vínculo entre las alcaldías mayores y las encomiendas, convirtiendo a las segundas en una forma de compensación ante la ausencia de salarios en las primeras (Hidalgo, 2001, pp. 83-84, 85, 86-87). Más aún, en el caso de la administración de encomiendas públicas, o de la puesta en práctica de obras de importancia estratégica, como la construcción naval, los alcaldes mayores eran los encargados de colectar el pago de tributos, o de administrar la compra de insumos (Alonso, 2003, pp. 63-70). Finalmente, la inexistencia de una economía agraria desarrollada o de una industria minera comparables a las de Nueva España y el Perú, hizo que la obtención y explotación de una encomienda fuese la principal forma de obtener recursos para participar en la principal actividad económica de los colonos españoles: el tráfico comercial en los galeones de Manila-Acapulco (Alonso, 2011, pp. 183-185).

Es importante recordar que esta infraestructura de movilización de recursos funcionaba en estrecha interacción con las estructuras de poder existentes en las sociedades indígenas (Scott, 1995, pp. 127-146). En efecto, los alcaldes mayores o los encomenderos trataban directamente con caciques regionales denominados gobernadorcillos, quienes tenían potestad sobre los pueblos de indios, generados por la misma administración española al concentrar los tradicionales núcleos tribales de determinadas zonas. Estos núcleos eran denominados barangays, y se hallaban compuestos por grupos de aproximadamente 30 a 100 familias. Sus caciques recibían el nombre de datus o cabezas de barangay. Estos eran los responsables de la extracción del tributo o trabajo de las familias bajo su autoridad, las cuales, a su vez, podían librarse enviando un sustituto en lugar del trabajador movilizado, con frecuencia, un esclavo temporal - obtenido por endeudamiento - o un esclavo permanente - usualmente un cautivo de guerra (Hidalgo, 2001, pp. 39, y p. 86) - La práctica de enviar un sustituto para los polos o elas era denominada opa, e implicaba un pago de seis, siete o hasta diez pesos a la tesorería real por parte del trabajador opado. Los primeros encargados de colectar esta cuota era los datus, o cabezas de barangay, quienes regularmente exigían al trabajador una compensación por ello (Phelan, 1959, p. 99). 
La interacción de ambos sistemas para la movilización de recursos, el español y el indígena, podía ser particularmente gravosa cuando las obras públicas de gran envergadura, como la construcción de un galeón, quedaban concentradas en una sola provincia. Más aún, los salarios a los trabajadores de polos y elas solían ser muy bajos: de cuatro a cinco reales al mes, cuando el costo de la vida requería de al menos cuarenta. Resulta evidente que las raciones de arroz, otorgadas como parte del sistema desde las primeras ordenanzas de gobierno, poco podían hacer para suavizar la dureza de este trato (Hidalgo, 2001, pp. 86-87). Más aún, los pagos de salarios a los trabajadores indígenas solían retrasarse sistemáticamente, durante años, generando así una deuda pública sobre las tesorerías reales que llegó a alcanzar 300.000 pesos en 1610 , y 1.000 .000 para 1619. Es probable que, ante los bajos salarios estipulados para los polos, la mayor parte de estas cantidades correspondiese a vandalas no pagadas (Phelan, 1959, p. 100).

De este modo, la industria de la construcción naval dependía directamente de la interacción administrativa de las alcaldías mayores con las encomiendas, privadas y públicas, para la obtención de los insumos navales producidos en las islas y, en especial, también, para la movilización de mano de obra indígena con destino a los cortes y arrastres de maderas, o para la realización de trabajos no calificados en el astillero. Es importante recordar que la principal actividad económica de la colonia española, el comercio con Nueva España, al igual que las operaciones de defensa y de ofensa contra las poblaciones musulmanas de Mindanao (y en su momento, contra los rivales ingleses y holandeses), dependían directamente de la construcción naval. En otras palabras, la principal actividad industrial de las islas, la construcción naval, no sólo estaba inextricablemente ligada a la supervivencia de la colonia española, sino que representaba, de hecho, la condición misma de su existencia.

\section{El papel de las órdenes religiosas, la legislación de esquipazones y cortes madereros, y la posibilidad de subcontratar la construcción naval en las regiones asiáticas. Reflexión final}

El mero énfasis en las manifestaciones de entusiasmo ante la construcción naval filipina durante su etapa formativa no déjà de ser una visión parcial, que obscurece tanto la dimensión de los perjuicios que ocasionaba a las sociedades indígenas como sus implicaciones en la posterior percepción de la calidad de los navíos construidos en las islas. Esta situación comenzó a ser denunciada por las órdenes religiosas allí desplegadas, dando lugar así a un progresivo enfrentamiento con los oficiales de la corona, que llegó a constituir un vector crucial de la historia de la construcción naval en el archipiélago. 
La primera orden en establecerse fue la de los agustinos, llegados con la expedición de Legazpi/Urdaneta, quienes fundaron en Luzón y parte de las Visayas la llamada provincia del Santísimo Nombre de Jesús. En 1578, los franciscanos crearon la provincia de San Gregorio, alrededor de la bahía de Manila, Laguna de Bay y Camarines, también en Luzón. Los jesuitas se establecieron en las Visayas en 1581, ocupando Cebú, Bohol, Negros, Panay, Leyte y Samar. En 1587 los dominicos se establecieron en Cagayán, Batán y Pangasinán, al norte de Luzón, en la llamada provincia del Santísimo Rosario. Finalmente, a partir de 1606, los recoletos de San Agustín fundaron la provincia de San Nicolás de Tolentino en zonas más distantes, como Zambales, Batán, Pangasinán Palawan y Mindanao, desde 1606 (Phelan, 1959, p. 31-32; Elizalde, 2015, p. 192). En 1579 se creó el primer obispado de Manila, dependiente de la Nueva España, el cual fue convertido en arzobispado independiente en 1595, con tres nuevos obispados creados ese mismo año en Cebú, Cagayán y Camarines (Elizalde, 2015, p. 191).

La presencia de las órdenes religiosas hizo posible la colonización del archipiélago, pues, además de su labor evangelizadora, los frailes se convirtieron en la espina dorsal del sistema económico de la colonia española. En efecto, además de atraer a las poblaciones locales por medio de la conversión, los frailes intervenían en la vida productiva de sus parroquias: desde el fomento de la agricultura a la construcción de edificios y obras públicas; y desde la gestión de obras pías, a la comercialización de determinados productos para el tráfico de Nueva España o al patrocinio de otras vías de exportación (Elizalde, 2015, p. 195). Finalmente, contribuían también a la fiscalidad pública, al interactuar con las poblaciones indígenas para el cobro de tributos y vandalas de diverso orden.

En 1582, el obispo de Manila, Fray Domingo de Salazar, denunció que las ordenanzas de gobierno promulgadas por Sande no se cumplían en absoluto, sobre todo en los puntos concernientes a la protección de los indígenas, «ni [...] cédula, ni providencia, ni ordenanza que esté dada para probecho y amparo de los yndios se guarda ni haçe caso della». Igualmente, refirió los perniciosos efectos de las esquipazones, los polos y las elas, en los siguientes términos:

es enviar á sacar los yndios que son menester para remar en una galera ó fregata [...] sin piedad ni respecto christiano [...] sin mirar si es casado ni soltero, ni si tiene á su muger mala ó los hijos, sino arreo, los lleva todos; y a acontecido traerle á la muger el marido y venir ella la barriga á la boca llorando que se lo dexen; que no tiene quien mire por ella, y dalle de palos que se vaya, y al pobre marido hacele yr mal que le pese; á otros aconteçe dexar á sus mugeres á la muerte y haçer ir a los maridos á remar, y los echan con hierros en las galeras y los açotan como si fueran forçados ó sentençiados, y la paga que les dan es muy poca; que solamente dan á 
cada uno por un mes quatro reales; y esto tan mal pagado, que los más se quedan sin ello y los pueblosde donde los sacan reparten entre sí la paga ó la dan á los que sacan para el remo; y esto es cosa muy çierta y averigüada [...] Lo mesmo pasa al cortar de la madera. Que haçen trabaxar á los yndios en cosas del servicio de Vuestra Magestad y les pagan poco, mal y tarde, y muchas veçes se quedan sin la paga.

Casi desde el inicio mismo de la ocupación española del archipiélago, la corona buscó contener los catastróficos efectos sociales de la construcción naval por medio de la legislación. De hecho, el primer reglamento contra la boga forzosa, establecido por Real Cédula en 1574, precede incluso a las primeras ordenanzas de gobierno promulgadas por Sande. Por este medio se prohibió la imposición a los indígenas de esquipazones que implicaran el traslado de una isla a otra, estableciendo a un mismo tiempo el pago de salarios. Claramente, estas órdenes no fueron respetadas, pues el Sínodo de Manila convocado en 1582 dedicó un apartado completo al trabajo forzado, incluyendo en él no sólo esta práctica, sino también los cortes de maderas (Cummins y Cushner, 1974, p. 121). En 1592, la corona prohibió el reclutamiento indígena para esquipazones, reservando esta actividad para la población china (Hidalgo, 2009b, p. 233, 235).

En 1607, el Consejo de Indias expidió una Real Cédula exigiendo justificar el creciente costo de la construcción de navíos y asegurar el pago de salarios a los trabajadores indígenas (McCarthy, 1995, p. 166; Sales-Colín, 2013, p. 68). Dos años después, en 1609, el Consejo despachó instrucciones precisas sobre la construcción naval al gobernador Juan de Silva, las cuales constituyeron la totalidad de la legislación dedicada a los cortes madereros hasta el advenimiento de las reformas borbónicas, en el siglo XVIII. Se trata de la Real Cédula de 26 de mayo de 1609, la cual ordenaba que se especificara si el tributo se cobraba en dinero o en especie, además de prohibir que la incapacidad de pagarlo condujese a la imposición de servicios laborales, constituyendo por tanto una especie de esclavitud por deudas (Cummins y Cushner, 1974, p. 198). Al respecto de la construcción naval, se prestaba atención a la configuración técnica de galeras, caracoas, y embarcaciones de cabotaje, señalando que los buques debían construirse siempre con las cubiertas por encima de los bancos de remeros, para reducir así su exposición a los elementos. La real cédula confirmaba también la prohibición de forzar los naturales a la boga, convirtiendo, en cambio, la práctica de las esquipazones en trabajo asalariado, preferentemente reservado para chinos y japoneses. La confirmación de esta medida es importante, visto que esta diferenciación étnico-laboral se extendió después explícitamente a los cortes de maderas, de la forma siguiente:

AGI, Filipinas, 6, r. 10, n. 180. 
mando, que no se repartan indios en más, ni menos número para grangerías particulares, ni públicas; pues a los cortes de maderas, navegaciones de Caracoas, y otras fábricas de esta calidad, en que está interesada mi Real Hacienda, y la pública comodidad de estas provincias, llevareys alquilados chinos, y iapones, que a la sazón se hallaren en Manila, y según estoy informado, avrá entre ellos suficiente número de jornales que vayan a estos ministerios, si les dan el justo jornal de su trabajo: por lo menos usareys de aquellos, que quisieren alquilarse, por excusar el concurrente número de indios; y en caso de que no se pueda quitar el repartimiento $[\ldots]$ y puesto que los chinos y japones no quieran; o no puedan satisfacer la urgencia y necesidad de estas obras públicas, areys diligencia con los indios para que acudan a ellas libre y voluntariamente, usando de los medios, que os parecieren convenientes a este efecto ${ }^{8}$.

Por otra parte, esta cédula contiene la clave para comprender la naturaleza de los problemas sociales generados por la construcción naval filipina durante el resto del siglo XVII: el permiso explícito, dentro del mismo texto, para continuar utilizando el trabajo forzado de las poblaciones indígenas en las esquipazones, las elas y los trabajos de astillero en casos de urgencia, y sólo para esas necesidades:

pero dado que aya falta de obreros voluntarios, permiso que se compelan algunos indios para trabajar en estas ocupaciones, con las condiciones, que se siguen, y no de otra manera. Que este repartimiento no se aga sino para cosas muy forzosas, y que no se puedan escusar pues en materias tan odiosas no a de bastar el mayor beneficio de mi Real Hacienda, o más comodidad de la república; y todo lo que no fuere preciso para su conservación; pesa menos, que la libertad de los indios?

La implicación es evidente: la corona prohibió el repartimiento en todos los casos, menos en los de urgencia o de interés estratégico. Sin embargo, como hemos señalado, la industria de la construcción naval caía, precisamente, en ambas categorías, pues la supervivencia misma de la colonia española dependía de ella. Dada la constante demanda de navíos, tanto para la guerra como para el comercio, el resultado de esta ambivalencia fue permitir, al mismo tiempo que se prohibía, precisamente la forma más intensiva, onerosa y frecuente del trabajo forzado indígena. Bajo esta luz, no resulta difícil comprender la notable frecuencia de las rebeliones acaecidas durante el período formativo de la industria: 1574, 1585, 1587, 1589, 1596, 1601 y 1607 (Prieto, 1984, pp. 144-145). A todo ello es preciso añadir los ataques de los holandeses, iniciados en 1600 (Boxer, 1985; Onrubia, 2011, pp. 264-267). Por otra parte, es preciso considerar las constantes incursiones de los moros de Mindanao, quienes, en

AGI, Filipinas, 329, II.

Ibidem. 
1617, atacaron directamente los astilleros, capturando a 400 obreros y matando a más de 200. Según el capitán de maestranza Sebastián de Pineda, en 1618 no quedaban más de 200 operarios indígenas en Cavite para el armamento de la flota de ese año ${ }^{10}$.

Este conjunto de factores tuvo efectos negativos sobre la industria de la construcción naval filipina, incidiendo en la percepción de ciertos sectores de la oficialidad española respecto a su eficiencia, e incluso a la conveniencia misma de mantenerla. Aquí se encuentra la clave para comprender los esfuerzos llevados a cabo durante los siglos XVII y XVIII para abandonar el fomento de los astilleros del archipiélago y subcontratar la producción de navíos en otros reinos asiáticos. Como ha señalado Sales-Colín, el gobernador Ronquillo presentó denuncias a propósito de la baja calidad de los navíos construidos en las islas en una fecha tan temprana como 1580. Según él, este problema era tan serio que los barcos que hicieron la carrera de Acapulco apenas sí pudieron cargar la mitad de su tonelaje en mercancías, además de llegar a ese puerto con el casco completamente podrido (Sales-Colín, 2013, p. 60).

De manera análoga, los frecuentes naufragios y pérdidas de galeones ocurridos durante toda la existencia de la carrera fueron con frecuencia atribuidos a su pésima hechura o al estado de su maderamen en el momento de iniciar el viaje. Una parte del problema residía en la escasez de maestros constructores de probada competencia, para lo cual su envío desde Vizcaya y Guipúzcoa representó una constante demanda en la correspondencia oficial de la gubernatura. Otro de esos problemas fue la ya mencionada presencia de técnicos chinos dirigiendo las labores de maestranza en los astilleros filipinos, una mano de obra que, junto a los otros trabajadores menos cualificados de ese mismo origen, compartía la desconfianza de las autoridades españolas. De hecho, en 1603, 1639 y 1662 tuvieron lugar sendas masacres de chinos en Manila y sus alrededores, las cuales contaron con la participación de la población indígena (Prieto, 1984, p. 145).

En palabras del gobernador Sebastián Hurtado de Corcuera: «las Naos todos los cuatro años se hazen como de nuebo por seis. Las maderas no duran más sin podrirse $\rangle^{11}$. Éste y otros ejemplos aislados, sin embargo, no deben inducir a error. Es posible señalar que la idea de que existió una polémica oficial en contra de la calidad de las maderas filipinas para favorecer la construcción en otros reinos asiáticos - como efectivamente sugirieron Hernando de los Ríos Coronel, Sebastián de Pineda y, posteriormente, los gobernadores Juan Niño de Távora, Sebastián Hurtado de Corcuera y Diego Fajardo - tiene algo de exageración ${ }^{12}$. La correspondencia oficial relacio-

AGI, Filipinas, 200, n. 206.

AGI, Filipinas, 8. r. 3, n. 63.

Blair y Roberston, 1904, vol. XVIII. AGI, Filipinas, 200, n. 206; 8, r. 1, n. 17 y r. 3, n. 63; 9, r. 1, n. 13. 
nada con estas propuestas de transferencia señala ocasionalmente la corta duración de los buques construidos en Filipinas, pero el argumento central es siempre la falta de caudales y el alivio de la población indígena, sumados, uno y otro, a la calidad y bajo costo de los insumos náuticos en otros reinos asiáticos. En el mismo sentido, es preciso considerar que las menciones al pudrimiento del casco de navíos y galeras era frecuente cuando se solicitaban recursos para construir otros nuevos, para lo cual representaba un argumento siempre conveniente. En otras palabras, es altamente probable que los defectos señalados fuesen resultado de unas condiciones coyunturales, y no necesariamente el reflejo de una generalizada percepción oficial respecto a la mala calidad de las maderas filipinas.

Dada la permanencia de la industria forestal en el archipiélago, la cual fue ampliamente desarrollada por el gobierno español durante el siglo XIX, y por la administración estadounidense después de 1898, es posible concluir que el problema no se hallaba realmente en la calidad de las maderas, ni siquiera en la percepción negativa que de ella pudiesen tener algunos oficiales, sino en la constante demanda de recursos financieros y materiales de la Real Hacienda, así como en las múltiples calamidades sociales derivadas de las distintas campañas de construcción naval. Ambos argumentos ha de situarse en el contexto de los mencionados intentos por transferir la fabricación de navíos al extranjero, y hay que considerar la posibilidad de que hubiesen sido formulados con la finalidad añadida de ejercer un control directo, y tal vez menos supervisado, de los recursos destinados a la fábrica de navíos. De igual forma, es preciso considerar el punto que posiblemente sea más importante: las propuestas de transferencia tuvieron lugar en el marco de un incremento de la presión por parte de la corona y las órdenes religiosas para poner fin a la explotación de los indígenas y, en determinados casos, también, a las rebeliones locales.

La compleja relación entre las órdenes religiosas y el funcionamiento económico de la presencia española en Filipinas hizo que los frailes se convirtiesen en protagonistas directos del desarrollo de la construcción naval. En efecto, éstos no sólo representaban un componente estructural de la movilización de recursos materiales y humanos, sino que también buscaron operar como un contrapeso a la explotación ejercida por la industria de la construcción naval. Existen casos documentados de sacerdotes protegiendo a las poblaciones indígenas de los alcaldes mayores y los encomenderos, hasta el punto de prender fuego a las forestas con el objetivo de impedir una campaña de cortes madereros (Wing, 2015, p. 156). Se trata, en todo caso, de una relación polifacética, pues algunas órdenes religiosas, particularmente los agustinos, recibieron acusaciones gubernamentales de contarse entre las causas de las desventuras de los nativos. El caso más importante es el del Discurso Parenético, del oidor Salvador Gómez de Espinosa (1657), el cual, en concordancia con el espíritu de la 
obra de fray Bartolomé de Las Casas, presentó serias denuncias contra la naturaleza de la movilización de recursos, no sólo por parte de la administración civil, sino también de las órdenes religiosas desplegadas en Filipinas (Cummins y Cushner, 1974, pp. 117-148). Dentro de estas denuncias se cuentan las esquipazones y las elas, pero, desde el punto de vista de la construcción naval, es preciso señalar que el texto se enfoca, tal como las denuncias de los frailes, sobre los excesos de la administración gubernamental, en esos dos rubros, y no en la incidencia, positiva o negativa, de las órdenes religiosas en ellos. 


\section{Bibliografía}

Alonso, Luis (2001), «La inviabilidad de la hacienda asiática. Coacción y mercado en la formación del modelo colonial en las islas Filipinas, 1565-1595», en Elizalde, María Dolores, Fradera Josep y Alonso, Luis (eds.), Imperios y naciones en el Pacifico, Madrid, CSIC, pp. 181-206.

Alonso, Luis (2003), «Financing the Empire: The Nature of the Tax in the Philippines, 1565-1804», Philippine Studies, 51, pp. 63-95.

Amaya, Sebastián (2017), «Poderío naval en las Indias: las galeras de Cartagena y Manila (1571-1621)», Revista de Estudios en Seguridad Internacional, vol. 3, 2, pp. 169-188. https://doi.org/10.18847/1.6.10

Argensola, Leonardo de (1609), Conquista de las islas Molucas, Madrid, Eduardo Martín.

Bankoff, Greg (2004), "'The Tree as the Enemy of Man': Changing Attitudes to the Forests of the Philippines, 1565-1898», Philippine Studies, vol. 52, 3, pp. 320-344.

Barrantes Moreno, Vicente (2004), Guerras piráticas de Filipinas (1570-1806), Málaga, Algazara, 2004.

Blair, Helen, y Robertson Alexander (1904), The Philippine Islands, 1493-1898, Cleveland, The Arthur and Clark Company, vol. XVIII.

Boxer, Charles (1985), «War and Trade in the Indian Ocean and the South China Sea, 1600-1650», Mariner's Mirror, 71, pp. 417-436. https://doi.org/10.1080/002533 59.1985.10656052

BRAND, Donald (1956), «The Development of the Pacific Coast Ports during the Spanish colonial Period in Mexico», en Comas, Juan (ed.) Estudios antropológicos publicados en honor al Doctor Manuel Gamio, México, UNAM, pp. 577-591.

Cervera, José Antonio (2013), «Los planes españoles para conquistar China a través de Nueva España y Centroamérica en el siglo XVI», Cuadernos Intercambio, vol. 10, 12, pp. 207-243.

Clayton, Lawrence (1980), Caulkers and Carpenters in a New World: The Shipyards of Colonial Guayaquil, Athens, University Center for International Studies.

Cordero Paciente, A. (2001), A Dictionary of Philippine Plant Names, Makati, The Bookmark Inc.

Cruz, Eugenio de la (1963), Lexicon of Philippine Trees, Manila, College Forest Research Institute.

Cummins, James y Cushner, Nicolas (1974), «Labor in the Colonial Philippines. The Discurso Parenético of Gómez de Espinosa», Philippine Studies, 22, p. 117-203. 
ChIrino, Pedro de (1890), Relación de las Islas Filipinas y de lo que en ellas han trabajado los padres de la Compañía de Jesús, Manila, Esteban Balbás.

Elizalde, María Dolores y Huetz De Lemps, Xavier (2015), «Un singular modelo colonizador: las órdenes religiosas en la administración española de Filipinas, siglos XVI al XIX», Iles i Imperis, vol. 17, 3, pp. 185-220.

Escalante Alvarado, García (2015), Viaje a las islas del Poniente, Santander, Universidad de Cantabria.

FADriquela, Cheek Sangalang (2013), Kahoy: Wood in the Philippines. Wood and its uses from Pre-Hispanic to Spanish Colonial Philippines, Manila, University of Santo Tomas Publishing House, 2. vols.

FernándeZ de Oviedo, Gonzalo (2006), General Historia de las Indias, Valladolid, Francisco Fernández de Córdoba, vol. II.

FLYNN, Dennis (2006), «Globalization Began in 1571», en Gills, Barry K. y William Thompson, Globalization and Global History, Nueva York, Routldge, pp. 232-247.

GARCÍA, María Fernanda (1992), «Galeones españoles y trabajo asiático. Un caso de comparación de recursos coloniales», Signos, II, pp. 47-69.

Giraldez, Arturo (2015), The Age of Trade: The Manila Galleons and the Dawn of the Global Economy, Londres y Nueva York, Rowman and Littlefield.

Hidalgo Nuchera, Patricio (2001), La recta administración. Primeros tiempos de la colonización hispana en Filipinas: la situación de la población nativa, Madrid, Polifemo.

Hidalgo Nuchera, Patricio (2009a), Una corografía ilustrada inédita: la descripción de las Yslas Philipinas de la Real Academia de la Historia de Madrid, León, Universidad de León.

Hidalgo Nuchera, Patricio (2009b), Encomienda, trabajo y tributo en Filipinas (1570-1608), Madrid, Polifemo.

Mallari, Francisco (1989), «The Spanish Navy in the Philippines, 1589-1787», Philippine Studies. Historical and Etnographic Viewpoints, vol. 37, 4, pp. 384-411.

Manguin, Pierre-Yves (2012), «Asian Ship-Building Traditions in the Indian Ocean at the Dawn of European Expansion», en Prakash, Om, The Trading World of the Indian Ocean, 1500-1800, Dehli, Pearson, pp. 597-629.

MARTín OnRubia, Miguel (2011), «La ofensiva naval neerlandesa sobre Filipinas en el contexto de la guerra de los Ochenta Años y su analogía con la llevada a cabo en los territorios americanos de la monarquía hispánica», en Manchado López, Marta y Luque, Miguel, Fronteras del mundo hispánico: Filipinas en el contexto de las regiones liminares novohispanas, Córdoba, Universidad de Córdoba. pp. 255-280. 
Martínez Shaw, Carlos (2001), «La exploración española del Pacífico en los tiempos modernos», en Elizalde, María Dolores; Fradera, Josep y Alonso, Luis (eds.), Imperios y naciones en el Pacifico, Madrid, CSIC, pp. 3-26.

McCarthy, William (1995), «The Yards at Cavite; Shipbuilding in early Colonial Times», International Journal of Maritime History, vol. 7, 9, pp. 149-162. https:// doi.org/10.1177/084387149500700208.

Morga, Antonio de (1997), Sucesos de las Islas Filipinas, Madrid, Polifemo.

OrtuÑo, José María (2005), «La expedición de Ruy López de Villalobos a las islas del Mar del Sur y del poniente», Anales de Derecho, 23, pp. 249-292.

OstolazA, María Isabel (2006), «Fray Martín de Rada, evangelizador, cosmógrafo y embajador en China», Revista Huarte de San Juan, Geografia e Historia, 13, pp. 177-198.

Peterson, Andrew (2014), Making the first Global Trade Route: The Southeast Asian Foundations of the Acapulco Manila Galleon Trade (1519-1650), Manoa, University of Hawaii.

Phelan, John Leddy (1959), The Hispanization of the Philippines, Madison, The University of Wisconsin Press.

Pigafetta, Antonio de (2004), Primer viaje en torno del globo, Madrid, Espasa.

Prieto, Ana María (1984), Filipinas durante el gobierno de Manrique de Lara, 1653-1663, Córdoba, CSIC.

Prieto, Ana María (1993), El contacto hispano-indígena en Filipinas según la historiografía de los siglos XVI y XVII, Córdoba, Servicio de Publicaciones.

Radell, David R., y PArsons, James J. (1971), «El Realejo: A Forgotten Colonial Port and Shipbuilding Center in Nicaragua», The Hispanic American Historical Review, 51, pp. 295-312. https://doi.org/10.2307/2512477.

Rojo, Justo (1997), Botanical Identification Handbook on Philippine Dipterocarps, Manila, Forest Products Research and Development Institute.

Rojo, Justo (1999), Revised Lexicon of Philippine Trees, Manila, Forest Products Research and Development Institute.

Rubio Sánchez, Manuel (1977), Historia del Puerto de la Santísima Trinidad de Sonsonate o Acajulta, San Salvador, Editorial Universitaria.

SAlEs-Colín Kortajerena, Ostwald (2013), «Polistas y arquitectura naval: una polémica contra los recursos de la tierra filipina durante el siglo XVII», Revista de Historia Naval, vol. 23, 122, pp. 59-76.

SAn Ignacio, Violeta (2001), «The State of the Philippine Economy during the Spanish Period», en Elizalde, María Dolores; Fradera, Josep y Alonso, Luis (eds.), Imperios y naciones en el Pacífico, Madrid, CSIC, pp. 381-394. 
SÁnchez Pedreño, José María (2001), «El triste final del Adelantado de la Mar del Sur, Vasco Núñez de Balboa», Anales de Derecho, 19, pp. 175-179.

Scott, William Henry (1982), «Boat Building and Seamanship in Classic Philippine Society», Philippine Studies, vol. 30, 3 (Third Quarter), pp. 335-376.

Scotr, William Henry (1995), Barangay: Sixteenth Century Philippine Culture and Society, Quezon City, Ateneo de Manila University Press.

Tamolang, F. (1995), «Strength Grouping Philippine Timbers for Various Uses», FPRDI Trade Bulletin Series, 4.

VALDEZ-BuBNov, Ivan (2017), «Comercio, guerra y tecnología: la construcción naval para la carrera de Filipinas (1577-1757)», en Rodríguez Hernández, Antonio José (ed.), Comercio, guerra y finanzas en una época en transición (siglos XVII-XVIII), Valladolid, Castilla Ediciones, pp. 225-270.

VIDAL, Sebastián (1874), Memoria sobre el ramo de montes, Madrid, Imprenta Estereotipia y Galvanoplastia de Aribau y C.

WING, John T. (2015), Roots of Empire. Forests and State Power in Early Modern Spain, c. 1500-1750, Leiden-Boston, Brill. https://doi.org/10.1163/9789004261372.

YEPES, Victoria (1996), Historia natural de las islas Visayas del Padre Alzina, Madrid, CSIC. 